\title{
INFECTIVITY TITRATIONS OF MYXOMA VIRUS IN THE RABBIT AND THE DEVELOPING CHICK EMBRYO
}

\author{
By FRANK FENNER \\ Department of Microbiology, John Curtin School of Medical Research, \\ Australian National University, Canberra \\ AND G. A. McINTYRE \\ Division of Mathematical Statistics, Commonwealth Scientific \\ and Industrial Research Organization, Canberra
}

During the intensive investigations of myxoma virus stimulated by the widespread epizootics of myxomatosis in Australia and Europe several strains of the virus which differ in pathogenic activity have been recognized (Fenner \& Marshall, 1956). Studies have been made of the relative transmissibility of several of these strains of virus by mosquitoes (Fenner, Day \& Woodroofe, 1956). The interpretation of these experiments depends to a considerable extent on the susceptibility of the rabbit skin to the different strains of myxoma virus, i.e. whether one viable virus particle deposited by a probing mosquito will initiate infection. The present experiments comprise a statistical study of this problem. Facilities for a direct comparison of total viral particles and infectious units are not yet available in Canberra. Since titration of myxoma virus on the chorioallantoic membrane is a cheaper and faster method of infectivity titration than the inoculation of rabbits, comparisons were made of the infectivities of the same series of preparations by titrations in rabbits and in eggs.

\section{MATERIALS AND METHODS}

Virus strains

Seven strains of myxoma virus were used: the Australian 'standard laboratory strain', a substrain of this, CAM 75, which had been passed 75 times on the chorioallantoic membrane, the Lausanne strain, KM13 (Myers, Marshall \& Fenner, 1954), two derivatives of the Uriarra strain, Uriarra III and Uriarra Contact (Mykytowycz, 1953), and Hurst's neuromyxoma (Hurst, 1937). Details of the origin of these strains and of their pathogenic behaviour in the rabbit is given elsewhere (Fenner \& Marshall, 1956).

Virus suspensions for titration were prepared from skin lesion material of diseased rabbits, and stored in large pools in glass ampoules at $-70^{\circ} \mathrm{C}$. The approximate titre of each pool was determined by titration of one ampoule in eggs.

\section{Experimental animals}

Rabbits from the University Breeding Establishment were used at the age of 4-5 months. They were inoculated intradermally in marked sites on the skin of the closely shaved back with $0 \cdot 1 \mathrm{ml}$. doses of virus, each animal receiving about one 
hundred inoculations. Readings for the appearance of skin lesions at the inoculation sites were made $3,4,5$ and occasionally 6 days after inoculation.

Chick embryos were inoculated on the chorioallantoic membrane after 11-12 days' incubation at $39^{\circ} \mathrm{C}$, the inoculum being spread on the chorion at the time of dropping that membrane (Beveridge \& Burnet, 1946). They were subsequently incubated for a further 3 days at $35^{\circ} \mathrm{C}$. before the inoculated portion of the membrane was removed, washed in formol saline, and examined with a binocular dissecting microscope. The numbers of specific pocks on the membranes were counted.

\section{Preparations of dilutions}

Dilutions of virus suspensions were made in cold gelatin saline (calciummagnesium saline containing $0.5 \%$ purified gelatin, and buffered to $\mathrm{pH} 7.2$ with borate buffer). Tenfold dilutions were made with $1 \mathrm{ml}$. volumes into $9 \mathrm{ml}$. blanks, different pipettes being used for each dilution. Twofold dilutions were made with $5 \mathrm{ml}$. volumes, using different pipettes for each dilution.

\section{EXPERIMENTAL RESULTS}

\section{Pock counts on the chorioallantois with closely spaced dilutions of several strains of myxoma virus}

Having determined the approximate pock counts with suspensions of different strains of virus, volumes of $1 \mathrm{ml}$. of the appropriate initial dilutions of different strains were added to volumes of $1,2,3,4$ and $5 \mathrm{ml}$. of diluent, all tubes being kept in an ice-cold bath. With each of these dilutions ten 11-day-old eggs were inoculated chorioallantoically, incubated at $35^{\circ} \mathrm{C}$. for 3 days, and pocks were counted. The results obtained in two experiments with the standard laboratory strain of virus are shown in Table 1. Comparable results were obtained with five other strains of virus, and the mean counts and their standard errors are set out in Table 2.

In each series the mean counts and corresponding standard errors at the various dilutions were multiplied by the dilution factor so as to make them directly comparable with one another (Table 3). These values for each series were further adjusted by a factor so as to make the mean count equal to 100 and in addition summary means with corresponding standard errors were computed for the values at each dilution.

A test was made of homogeneity of adjusted counts within each series and also for the summary values (Welch, 1951). Only in the series 'Neuromyxoma expt. I' was the heterogeneity significant at $P<0.05$, and in this series there is no evidence of consistent trend. The significance level for the summary values is $P=0 \cdot 10$ with a slight suggestion of lower counts at the higher dilution than would be expected. Tentatively one would infer that counts decrease proportionally to the dilution, at least over the range considered here.

\section{Titration of several strains of myxoma virus by the intradermal inoculation of rabbits}

Parker (1940) studied the titration of myxoma virus by the intradermal inoculation of rabbits, and concluded that the response curve was consistent with the hypothesis that one virus particle could initiate infection. We wished to know 

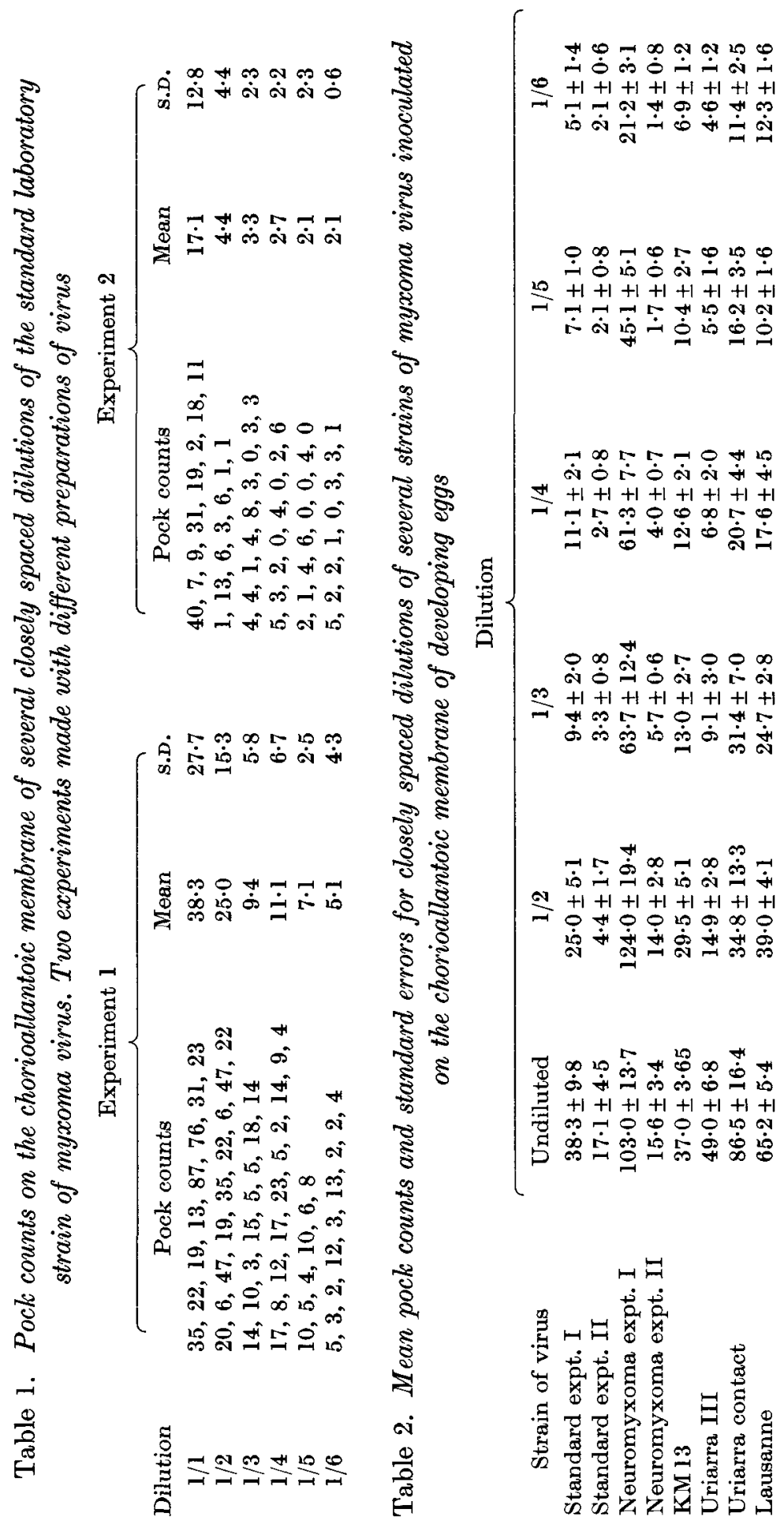
whether this was true for several strains of myxoma virus possessing different degrees of virulence for the domestic rabbit, and also to know whether variations in susceptibility occurred between rabbits.

Four rabbits were used for each of six strains of myxoma virus. The backs were shaved and marked in a $12 \times 8$ checkerboard. Serial twofold dilutions were inoculated intradermally in volumes of $0.1 \mathrm{ml}$., each dilution being inoculated in eight sites. In two rabbits the most dilute suspensions were inoculated at the head end of the rabbits, and in the other two at the tail end. The detailed results of the titration of the standard laboratory strain of virus are shown in Table 4. The summarized results for the six strains are set out in Table 5, which includes also the results of titrations of suitable dilutions of the same suspensions on the chorioallantoic membrane. The dilution of each preparation which would produce about fifty pocks on the chorioallantoic membrane with a $0.05 \mathrm{ml}$. inoculum has been designated in Table 5 by the negative logarithm ' $x$ ', and the dilutions used for inoculation of the other eggs, and of the rabbits, are related to this dilution.

Table 3. Adjusted mean counts for closely spaced dilutions of several strains of myxoma virus, inoculated on the chorioallantoic membrane of developing eggs

\begin{tabular}{lrrrrrr}
\multicolumn{1}{c}{ Strain of virus } & Undiluted & $1 / 2$ & $1 / 3$ & $1 / 4$ & $1 / 5$ & $1 / 6$ \\
Standard expt. I & 101 & 132 & 75 & 118 & $\mathbf{9 4}$ & 81 \\
Standard expt. II & 147 & 76 & 85 & 93 & 90 & 108 \\
Neuromyxoma expt. I & 54 & 131 & 101 & 129 & 119 & 67 \\
Neuromyxoma expt. II & 100 & 179 & 110 & 103 & 54 & 54 \\
KM 13 & 80 & 127 & 84 & 108 & 112 & 89 \\
Uriarra III & 156 & 95 & 87 & 87 & 88 & 88 \\
Uriarra contact & 108 & 87 & 117 & 103 & 101 & 85 \\
Lausanne & 95 & 113 & 108 & 102 & 74 & 107 \\
$\quad$ Mean & 105 & 118 & 96 & 105 & 91 & 85
\end{tabular}

The mean number of infections per dose at the highest dose level $(\lambda)$ was calculated for each rabbit from the tables of Fisher \& Yates (1953), and for each group of four rabbits. Comparison of the estimates of $\log \lambda$ in the individual rabbits of each group by the $\mathrm{F}$ test indicated that there was no significant 'between-rabbits' variation except with the two attenuated Australian field strains, Uriarra III and KM 13. For Uriarra III $F=3.157(P<0.05)$, and for KM $13 F=7.71(P<0.001)$. In the experiments performed with these two strains, therefore, there was evidence of differences in the susceptibility to infection (as evidenced by the production of a local skin lesion) between laboratory rabbits.

The data were then tested by Moran's test (Moran, 1954a,b) for their conformity with the exponential distribution. The results are shown in Table 6 .

With a single exception the deviates from the expected values are not significant at the $5 \%$ level, and the occurrence of this exception is in accordance with expectation. The evidence strongly suggests that within rabbits the change in proportion of failures with increasing dilution conforms with expectation for a twofold dilution series. This would be consistent with a constant probability of infection by a virus infective unit at all dilutions and stability of the infective unit with dilution. 


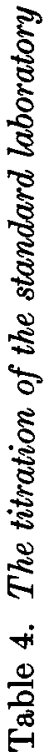

3

8

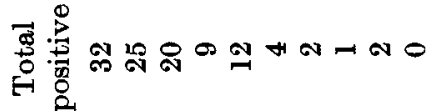

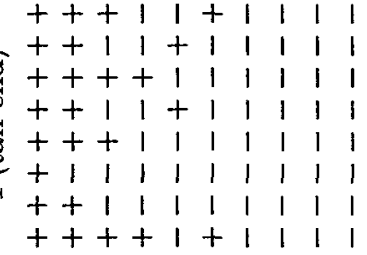

$++1+11111$

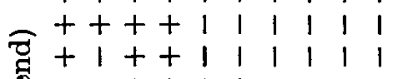

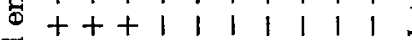

哥 $++11++1111$ $+++1111+1$

$\infty+11+111111$

$+1+1+11111$

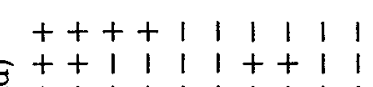
$\frac{g}{0}++11+11+1$ $+t+1 \quad 1 \quad 1 \quad 1 \quad 1111$ $\Phi+111+11111=$

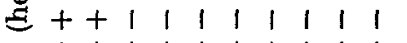
a $t++1+1 \quad 1 \quad 1 \quad 1 \quad 1$ $+t+1+1|1| 1$

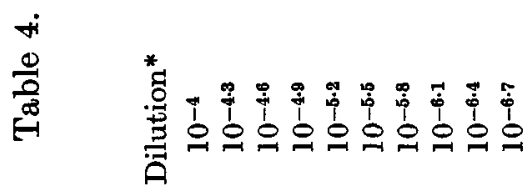

$++1 \quad 1 \quad 1 \quad 1 \quad 1 \quad 1111$ $\overline{8}+1+++11111$

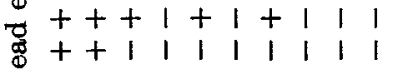
$\Xi+t+1 \quad 1 \quad 1 \quad 1 \quad 1 \quad 11$ $n+111+11111$ $+t++1 \quad 1 \quad 1 \quad 1 \quad 1$

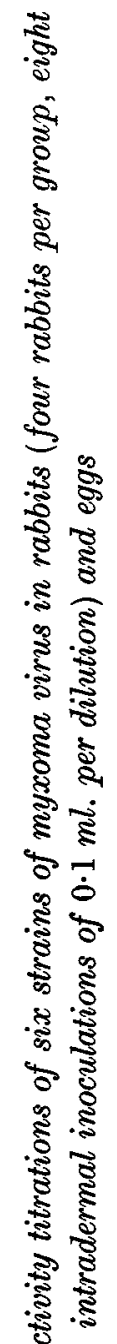
$\widehat{\bar{g}}+++1 \quad 1 \quad 1 \quad 1 \quad 1 \quad 1 \quad 1 \quad$

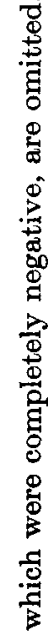

है 奌.

胥

is

$\frac{0}{6}$

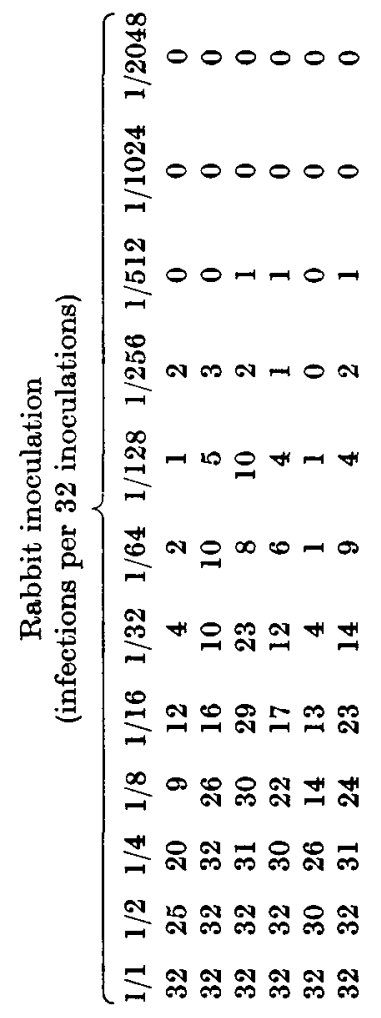

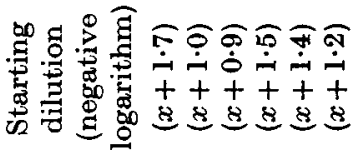

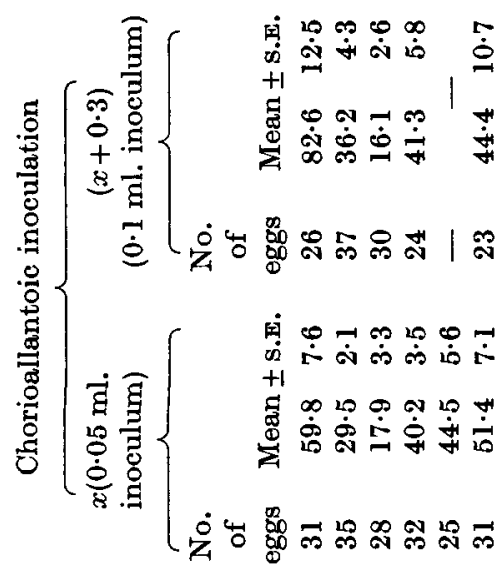

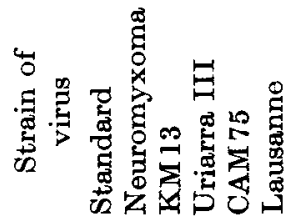


III. Titration of several strains of myxoma virus on the chorioallantoic membrane

The distribution of infectious particles in aliquots of a given suspension is Poissonian, and if every infectious particle produces a pock (just as in colony counts of bacteria every viable bacterium will produce a colony under suitable conditions), the distribution of pocks should also be Poissonian. In none of the eleven series of pock counts summarized in Table 5 does this occur. It was thought that, as Reid, Crawley \& Rhodes (1949) found with pock-counting titrations of fowlpox virus, this might be due to technical defects of the method of inoculation usually adopted, for example, the dropping of the membrane by the inoculum. However, in no case were the counts made by first dropping the membrane and then inoculating 0.05 or $0.1 \mathrm{ml}$. volumes on the dropped membrane (second column of Table 5) significantly different from those made by the other method. The variation seems to reside in the susceptibility of the eggs.

Table 6. Moran's tests for exponential distribution of infected sites in the infection of rabbits with different strains of myxoma virus. Twofold dilution steps, eight inoculation sites per dilution per rabbit, four rabbits per strain of virus

Test criterion $T=\Sigma f_{m}\left(n-f_{m}\right)$, where $f_{m}$ is the number sterile out of the number of inoculation sites at each dilution.

$$
\begin{array}{rlr}
\text { For } 8 \text { replicates: } & E(T)=56, & \text { For } 32 \text { replicates: } \\
\operatorname{var} T=144 \cdot 6, & \text { var } T=10,187, \\
\text { s.E. }=12 \cdot 02 . & \text { s.E. }=100 \cdot 96 .
\end{array}
$$

$\begin{array}{ccccccc}\text { Rabbit } & \overbrace{\text { CAM 75 }} & \text { KM 13 } & \text { Uriarra III } & \text { Lausanne } & \begin{array}{c}\text { Neuro- } \\ \text { myxoma }\end{array} \\ \text { no. } & \text { Standard } & \text { Strain of virus } \\ 1 & 62 & 59 & 29^{*} & 61 & 46 & 55 \\ 2 & 72 & 46 & 43 & 62 & 74 & 62 \\ 3 & 72 & 48 & 51 & 66 & 52 & 62 \\ 4 & 59 & 58 & 47 & 56 & 76 & 49 \\ \text { Composite } & 1125 & 889 & 888 & 1105 & 889 & 1074\end{array}$

* Significant at $5 \%$ level.

For all strains the distribution of pock counts is positively skew, with some counts near zero. If it is postulated that the distribution of pock counts arises from a varying probability $(p)$ of infection between eggs and a Poisson variation in the number of particles applied, then estimates of the coefficient of variation, and of $\beta_{1}$ and $\beta_{2}$ for the distribution of $p$ are obtainable from the cumulants of the frequency distribution of pock counts:

$$
\begin{aligned}
& \text { Expectation of } K_{2} \text { (pocks) }=m+m^{2} K_{2}(p) / \bar{p}^{2}, \\
& \text { Expectation of } K_{3} \text { (pocks) }=m+3 m^{2} K_{2}(p) / \bar{p}^{2}+m^{3} K_{3}(p) / \bar{p}^{3}, \\
& \text { Expectation of } K_{4} \text { (pocks) }=m+4 m^{2} K_{2}(p) / \bar{p}^{2}+6 m^{3} K_{3}(p) / \bar{p}^{3}+m^{4} K_{4}(p) / \bar{p}^{4},
\end{aligned}
$$

where $m$ is the population mean number of pocks of which an estimate is given by the sample. From these relations $K_{2}(p) / \bar{p}^{2}, K_{3}(p) / \bar{p}^{3}$ and $K_{4}(p) / \bar{p}^{4}$ can be estimated and hence $\beta_{1}(p)$ and $\beta_{2}(p)$. These measures place limits on the form of the distribution of $p$ which is probably similar to Type I of the Pearson system of curves but modified in the neighbourhood of zero and possibly in the upper tail. 
A rough indication of the upper bound to the mean value of the distribution of $p$ for these eggs is given by the reciprocal of the ratio of largest to mean count after decreasing it for Poisson effect on the range and increasing it for an increase of sample size from about 60 to infinity. The removal of the Poisson effect reduced the coefficient of variation by about $3 \%$, and the average effect on the range is not likely to be more than $5 \%$. The order of adjustment for sample size is suggested by the fact that the average ratio of largest to mean for the two subpopulations of each strain of about thirty eggs increased to $13 \%$ on pooling, in agreement with the increase in average range for two samples of thirty from a normal population on pooling. It is considered that the effect of increasing the sample from sixty to infinity for the finite distribution of $p$ is not likely to be less than the effect on the average range for a tenfold increase in the size of sample from a normal population, which gives a factor of $1 \cdot 33$. This procedure of extrapolation by analogy is frankly speculative but we consider the factor $\mathbf{1 . 3 3}$ to be a reasonable minimum. The joint adjustment factor is 1.28 and the values for the adjusted reciprocals are given in the last column of Table 7 .

The combined evidence over the several strains suggests that at most only about one out of four infective units produces a pock taken over a population of eggs as susceptible as those used in this experiment. It should be recorded that corresponding to a particular mean number of pocks the variance of counts for this batch of eggs was substantially higher than for an earlier series suggesting that between batches the distribution of $p$, and therefore mean $p$, can change.

This was borne out by other experiments (Marshall unpublished) in which it was necessary to titrate aliquots of a preparation of the standard laboratory strain of virus 36 times during a period of 9 months, during which time the virus was stored at $-70^{\circ} \mathrm{C}$. Between three and twenty eggs, usually four to six, were used for each titration. The logarithms of the counts were analysed for variation between eggs at single titrations and between titrations. The variance of the logarithms showed no trend with the titration means. There was clear evidence of a decrease in the means over the period $(P<0.001)$ accounted for wholly or almost wholly by a linear trend (fraction lost per day $=0 \cdot 0023$ ). Because an annual cycle was not completed it was not possible to attribute this to either a seasonal variation in egg susceptibility or progressive loss in potency of the preparation. However the variation of the means of titrations about the regression line was highly significant.

Taking the effects of variation between eggs within batches and between means of batches as multiplicative the estimated coefficient of variation between eggs within a batch was estimated to be $63 \%$, and the coefficient of variation of the mean of the batch about the regression line was $38 \%$. If the trend itself is due to seasonal variation in egg susceptibility then the coefficient of variation of batch means is of the order of $42 \%$.

Attempts to identify the source of variation between batches have so far been unsuccessful. Tests with 11-day-old eggs showed that the period of manipulation at room temperature during the drilling and inoculation of the eggs was without 


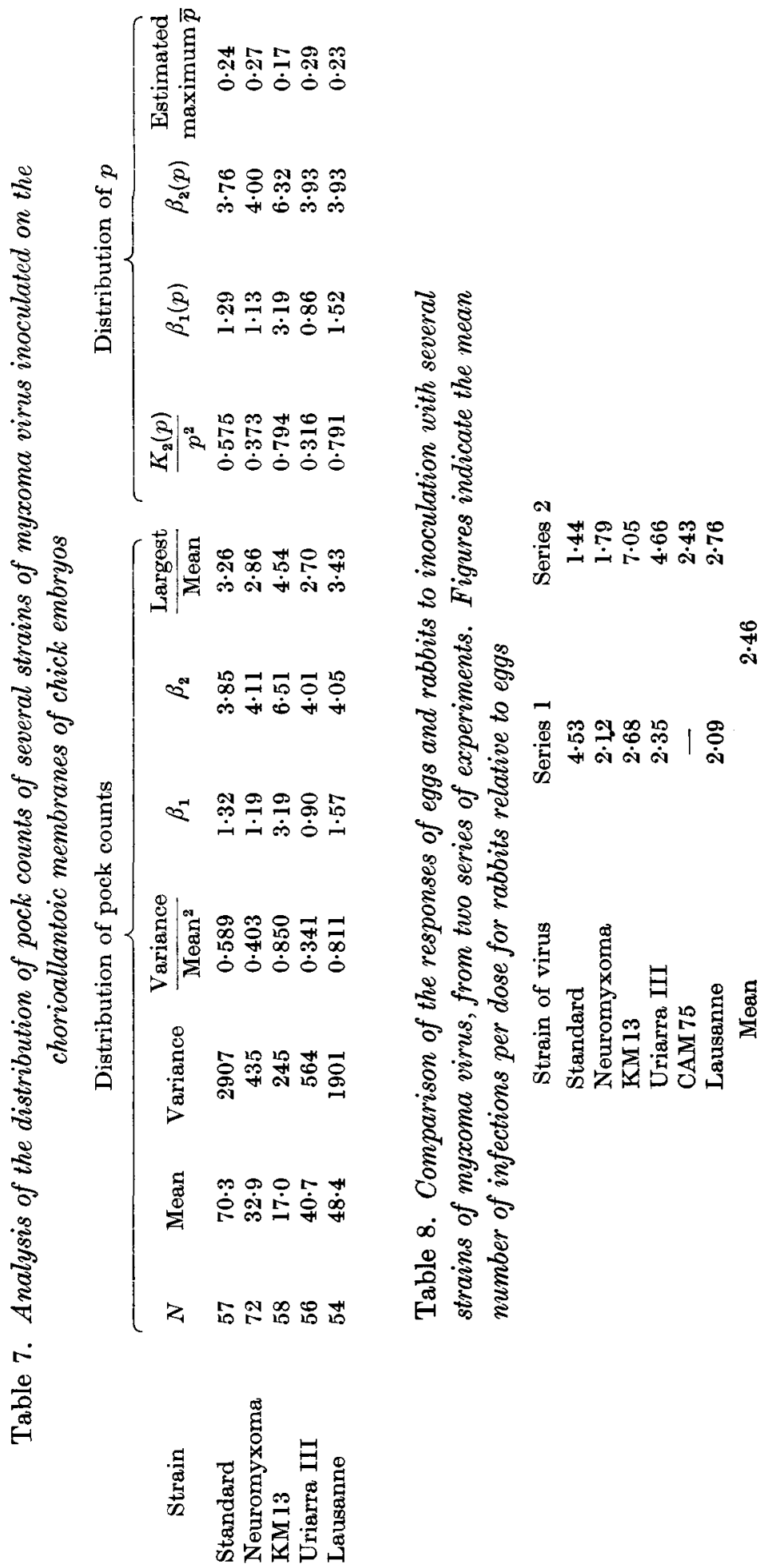


effect on the inter-egg variation or the mean pock count, at least within a range of 0-45 min. at room temperature.

\section{Relationship between titration on the chorioallantoic membrane and in the rabbit skin}

It remains to compare the responses to titration of the different strains of virus in the rabbit and the egg. To do this the mean number of infections per dose $(\lambda)$ for a particular dilution and volume has been estimated from the dilution titration on rabbits and the number of pocks for the same dilution and volume from the pooled egg counts. These estimates are compared in Table 8 . The results of an earlier series of experiments comparing egg and rabbit titrations of the same strains of virus are included in this table. The design differed somewhat from the experiments described in detail in this paper, in that two successive twofold dilutions were inoculated on each of four rabbits, each in twenty-five sites. The spacings of the dilutions chosen did not in every case secure a completely positive and completely negative result, so that Moran's test could not be applied. In addition, 'betweenrabbit' comparisons of susceptibility could not be determined. However, the data were as valid for estimation of comparative rabbit-egg titres as in the second experiment, and these are the figures included in Table 8, series 1 .

The rabbit skin was more sensitive than the chorioallantoic membrane with each strain of virus tested. Even passage of the virus on the chorioallantoic membrane for seventy-five consecutive passages failed to change the ratio.

Using log ratios and their corresponding variances the interaction of strains with series is highly significant, indicating the presence of some experimental factors not accounted for in determining the variances, such as egg batch variation within series, or a genuine interaction of strains and series. The estimate of variance between strains taken over the two series is less than the interaction variance, so that the evidence is not in conflict with the hypothesis that the ratio of estimate from egg and rabbit titrations is on the average the same with different strains of myxoma virus.

The weighted ratio over both series is $\mathbf{2} \cdot 46$. If each infective unit causes an infection in the rabbit this sets an upper limit to $\bar{p}$ for eggs of 0.41 . If, on the other hand, the upper limit to $\bar{p}$ of about 0.25 be accepted then the maximum value to the mean probability of causing an infection in the rabbit, over a population of laboratory rabbits as used in this experiment, is 0.61 . This is more in accordance with the facts since there is clear evidence that rabbits can differ in susceptibility.

\section{DISCUSSION}

Dulbecco \& Vogt (1954), working with poliomyelitis viruses which produced plaques on monolayer tissue cultures of monkey kidney epithelial cells, found that there was a proportional relationship between the numbers of plaques and the relative virus concentration over the small range used in their titrations, and they developed the statistical justification for the belief that such a linear relationship indicated that each plaque was initiated by a single infective unit. The variability in replicate counts of myxoma virus on eggs was greater than that observed by 
these workers, but with all strains there was a reasonably close approximation to proportionality between pock count and the virus dilution. It can be inferred, therefore, that a pock on the chorioallantoic membrane results from infection initiated by one infective unit, and that the infective unit is stable with dilution.

Parker (1940) carried out experiments on the titration of myxoma virus (the predecessor of our standard laboratory strain) by the intradermal inoculation of rabbits, and concluded that a single viral particle could initiate infection. He found in each of two experiments that there was a systematic tendency towards departure of the experimental from the theoretical curve in that at low dilutions there was a consistent excess of negative results, the reverse being true at high dilutions. It is not clear from the text how the inoculations of various dilutions were distributed between rabbits. However, variation in susceptibility between rabbits was evidently ignored as a factor and this could possibly account for the systematic departure from the expected curve.

We found no difference in the susceptibility of rabbits to different strains of virus, and the change in the proportion of failures with increasing dilution conformed with expectation for a twofold dilution series. Without making total counts of viral particles with the electron microscope it is impossible to decide what proportion of particles is able to initiate infection in the rabbit skin, but the data were consistent with a constant probability of infection by an infectious unit of the virus at all dilutions, and with stability of the unit with dilution.

Variation in the susceptibility of rabbits to infection was found with two of the strains-Uriarra III and KM 13. These two strains produce flat skin lesions, and gave rise to more difficulty than other strains in making a decision as to whether a lesion was due to inoculation at that site, or was an early secondary lesion. It is not possible to say whether the 'between-rabbit' variation found was due to this technical difficulty, or was due to chance allocation of very resistant or very susceptible rabbits to these groups. In other experiments we have confirmed the observation of Houlihan \& Derrick (1945) that rabbits in poor physical condition were much less likely than healthy rabbits to produce recognizable primary skin lesions after the intradermal inoculation of small doses of virus.

Houlihan \& Lawson (1945) were able to detect specific local lesions much less frequently when inoculations of similar doses of virus were made in the abdominal skin, than in the skin over the back and flanks. We found no significant differences in different parts of the shaved back.

Reid et al. (1949) found that the 'between-eggs' distribution of pocks on the chorioallantoic membrane, following the inoculation of fowlpox virus, did not differ greatly from the Poisson distribution. This was not the case with Herpes simplex (Scott, Coriell, Blank \& Gray, 1953), nor did we find this with any of the strains of myxoma virus examined.

There is evidently variation in the susceptibility of different chorioallantoic membranes to infection with myxoma virus. On the assumption that the distribution of pock counts is due to superimposing Poisson variation in number of particles applied on a probability distribution of susceptibility of eggs, it was possible from the statistics of the distribution of pocks to estimate the coefficient of 
variation, $\beta_{1}$ and $\beta_{2}$, of the distribution of infectivity. These indicate that the mean $p$ must be less than 0.5 . A rough estimate from the ratio of the largest count to the mean adjusted for Poisson variation and sample size suggests that the ratio of the maximum value to the mean is about $0 \cdot 25$. If this figure be accepted then the maximum value for average probability that an infectious unit will produce a lesion in the rabbit is of the order of $0 \cdot 6$.

Titration in eggs is much more efficient than titration in rabbits when dealing with suspensions of virus of unknown concentration, for results of much the same accuracy can be obtained by the use of six eggs at each of three tenfold dilutions as can be obtained by using several rabbits inoculated in many sites with twofold dilutions. Further, if the concentration of virus is higher than anticipated a reasonable estimate of the virus concentration can be made from membranes by counting pocks within a defined fraction of the total area, but only a minimum figure can be calculated from a titration in rabbits which fails to straddle the endpoint.

\section{SUMMARY}

Titrations of the same preparations of myxoma virus have been made on the chorioallantoic membranes of developing chick embryos and by the intradermal inoculation of rabbits. Six strains of widely differing virulence for the rabbit were used.

No differences were found in the infectivity of different strains for either the rabbit or the egg, even though the severity of the symptoms produced in the rabbit varied greatly with different strains; and although some had never previously been inoculated in eggs and one had been subjected to seventy-five serial passages on the chorioallantoic membrane.

The egg was less sensitive than the rabbit, the mean number of infections per dose for rabbits relative to eggs being $2 \cdot 46$, taken over all strains. This ratio did not differ significantly between strains of myxoma virus.

There was evidence of some host variation in rabbit susceptibility and a great deal of variation in egg susceptibility. The maximum value for the average probability that an infectious unit would produce a lesion in the rabbit skin was about $0 \cdot 6$, and the maximum value for the average probability that an infectious unit would produce a pock on the chorioallantoic membrane was $0 \cdot 25$.

Titration of several closely spaced dilutions of different strains of the virus on the chorioallantoic membrane showed a reasonably close approximation to a proportional relationship between the pock count and the virus dilution. The change with dilution in the proportion of failures to infect rabbits approximated to expectation. Both lines of evidence are consistent with the hypothesis that a pock or skin lesion is initiated by one infective unit of virus, and that the infective unit is stable with dilution. 


\section{REFERENCES}

Beveridge, W. I. B. \& Burnet, F. M. (1946). Spec. Rep. Ser. med. Res. Coun., Lond., no. 256.

Dulbecco, R. \& Vogt, M. (1954). J. exp. Med. 99, 167.

Fenner, F., DAY, M. F. \& Woodroofe, G. M. (1956). J. Hyg., Camb. (3rd paper of group). Fenner, F. \& Marshald, I. D. (1956). J. Hyg., Camb. (in preparation).

Fisher, R. A. \& Yates, F. (1953). Statistical Tables for Biological, Agricultural and Medical Research, p. 57. Edinburgh: Oliver and Boyd.

Houlihan, R. B. \& Derrick, W. A. (1945). Science, 101, 364.

Houlihan, R. B. \& Lawson, G. M. (1945). J. infect. Dis. 76, 40.

Hurst, E. W. (1937). Brit. J. exp. Path. 18, 15.

Moran, P. A. P. (1954a). J. Hyg., Camb., 52, 189.

Moran, P. A. P. (1954b). J. Hyg., Camb., 52, 444.

Myers, K., Marshall, I. D. \& Fenner, F. (1954). J. Hyg., Camb., 52, 337.

MyкYTowycz, R. (1953). Nature, Lond., 172, 448.

PARKer, R. F. (1940). J.exp. Med. 71, 439.

Reid, D. B. W., Crawley, J. F. \& Rhodes, A. J. (1949). J. Immunol. 63, 165.

SCotT, T. F. M., Corielt, L. L., Blank, H. \& GraY, A. (1953). J. Immunol. 71, 134.

WELCH, B. L. (1951). Biometrika, 38, 330.

(MS. received for publication 28. ХI. 55) 